\title{
BMJ Open Assessment of mental health literacy using a multifaceted measure among a Chinese rural population
}

Yu Yu, ${ }^{1}$ Zi-wei Liu, ${ }^{1} \mathrm{Mi} \mathrm{Hu},{ }^{1}$ Xi-guang Liu, ${ }^{1}$ Hui-ming Liu, ${ }^{1}$ Joyce P Yang, ${ }^{2}$ Liang Zhou, ${ }^{1}$ Shui-yuan Xiao ${ }^{1}$

To cite: Yu Y, Liu Z-wei, $\mathrm{Hu} \mathrm{M}$, et al. Assessment of mental health literacy using a multifaceted measure among a Chinese rural population. BMJ Open 2015;5:e009054. doi:10.1136/bmjopen-2015009054

- Prepublication history for this paper is available online. To view these files please visit the journal online (http://dx.doi.org/10.1136/ bmjopen-2015-009054).

Received 12 June 2015 Revised 22 August 2015 Accepted 25 August 2015

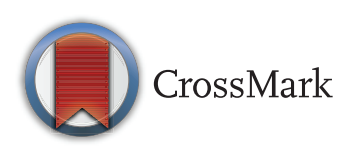

${ }^{1}$ Department of Social Medicine and Health Management, School of Public Health, Central South University, Changsha, Hunan, China

2Department of Psychology, University of Washington, Seattle, Washington, USA

Correspondence to Professor Shui-yuan Xiao; xiaosy@live.com

\section{ABSTRACT}

Objectives: The present study aims to assess mental health literacy (MHL) using a standardised multifaceted 20-item instrument called Mental Health Knowledge Questionnaire (MHKQ) developed by the Chinese Ministry of Health, among a rural Chinese population.

Setting: Four villages in Liuyang county of Hunan province, China.

Participants: This was a cross-sectional study. A multistage cluster-sampling method was adopted, leading to a final sampling frame of 2377 residents aged 18-60 years from four villages of Liuyang county. Included in the study were residents aged 18-60 years living in their village for at least half a year; excluded were those not living in the areas during the research period, those with difficulty in communication due to serious physical or mental illness and those who were cognitively impaired or actively psychotic. Finally, 2052 participants completed the survey.

Primary and secondary outcome measures:

Primary outcome was correct response rate of the MHKQ; secondary outcome measures were association between sociodemographics and $\mathrm{MHL}$, and association between MHL and health outcomes.

Results: Correct response rates for the $20 \mathrm{MHKQ}$ items ranged from $19 \%$ to $94 \%$, with a mean rate of $58 \%$. Younger age $(r=-0.02, p<0.01)$, higher education ( $r: 1.38-2.69, p<0.01)$ and higher income $(r=0.41$, $p<0.01)$, were independently associated with higher MHL. MHL was independently associated with self-rated general health $(r=2.31, p<0.01)$, depression $(r=-0.09, p<0.01)$ and anxiety $(r=-0.07, p<0.05)$.

Conclusions: MHL in the rural areas of Liuyang is lower than that reported in urban areas of China. There is much room for improvement with regard to $\mathrm{MHL}$ promotion in rural areas of China. Younger age, higher education and higher income are the three robust factors related to higher MHL, so cohort-specific educational intervention efforts may be indicated.

\section{INTRODUCTION}

Mental health literacy (MHL) refers to knowledge and beliefs about mental health disorders that aid in their recognition, management or prevention. ${ }^{1}$ First introduced in

\section{Strengths and limitations of this study}

- Broadening the definition of mental health literacy (MHL) to include 'illness literacy' as well as 'health literacy'.

- Assessing population level MHL in rural China using a multifaceted measurement tool-the Mental Health Knowledge Questionnaire (MHKQ) -instead of vignettes based on Western cultural contexts.

- Exploring the relationship between MHL and a range of health outcomes, which is a substantially underdeveloped area of research.

- The cross-sectional study design makes causal relationships undeterminable.

- Lack of comparison between MHKQ with other multifaceted scales intending to measure MHL as the MHKQ has not be utilised outside of China.

1997 by Australian researchers Jorm et al, this definition has become a gold standard within research and practice on MHL. ${ }^{2}$ According to Jorm et al, MHL includes seven key components: (1) the ability to recognise specific disorders; (2) knowledge of how to seek mental health information; (3) knowledge of risk factors of mental illness; (4) knowledge of causes of mental illness; (5) knowledge of selftreatments; (6) knowledge of professional help available and (7) attitudes that promote recognition and appropriate help-seeking. Based on Jorm et al's definition, O'Connor et $a l^{2}$ grouped the seven key components of MHL into recognition, knowledge of factors relating to mental health, and attitudes and beliefs about mental disorders. Of the various aspects of MHL, recognition of mental disorders is the most common attribute measured in MHL, as it is the first step towards MHL and forms a pre-requisite for measurement of other attributes.

Ever since the definition of MHL was first proposed, the concept has drawn extensive attention to the public's low level of MHL, 
with growing evidence showing low recognition rate of common mental disorders among various populations in developed and in developing countries. For example, the recognition rate of depression is $<50 \%$ among adolescents ${ }^{3}$ and college students ${ }^{4}$ in the USA, and is only $11 \%$ among patients with cancer and 25\% among lay people in Japan. ${ }^{5}$ One study among Portuguese youth reported the recognition rate of schizophrenia or psychosis as $42.17 \%$ and $22.21 \%$, respectively, ${ }^{6}$ while another study among resettled Iraqi refugees found that only $14.2 \%$ of participants recognised post-traumatic stress disorder. Further investigation of the factors related to MHL has found that female gender, ${ }^{7-9}$ higher education, ${ }^{10} 11$ younger age ${ }^{10-12}$ and being employed ${ }^{11}$ are associated with higher MHL. The importance of MHL in help-seeking behaviour has also been studied, with evidence indicating that lack of MHL is a main challenge that patients and families face in seeking help, ${ }^{13} 14$ especially in Asian cultures, where utilisation of mental health services is lower across the board. ${ }^{15}$ There is also growing evidence showing that higher MHL is associated with better access to and utilisation of mental health services. ${ }^{16}{ }^{17}$ Moreover, increasing MHL can improve helpseeking behaviour, the quality of mental health service and, as a result, patients' symptoms. ${ }^{18} 19$

Despite the rich and abundant research on MHL all over the world, it is important to note that there are three major limitations in existing studies on MHL.

First, the most widely used definition of MHL by Jorm is problematically narrow in its scope. Based on the Diagnostic and Statistical Manual of Mental Disorders (DSM-IV), Jorm's definition of MHL is illness-oriented, neglecting the fact that health-oriented literacy including self-regulation strategies and techniques to improve mental health should also be incorporated as an important component of MHL. ${ }^{20}$ Kusan $^{21}$ and Wei $e t a l^{22}$ have suggested a broader definition of MHL including knowledge to enhance and maintain good mental health, knowledge of mental disorders and their treatments, decreased stigma against mental disorders and enhanced help-seeking efficacy, however, this definition is rarely used in research related to MHL.

Second, existing assessment of MHL mainly relies on a vignette interview approach, which may not comprehensively reflect respondents' understanding of mental health. In the vignette interview approach, a vignette is presented that describes an individual with a mental health difficulty and asks a series of questions related to participants' understanding of what is 'wrong' with the individual in the vignette. ${ }^{23}$ The vignette interview approach usually targets one specific mental disorder without reflecting understanding of the multifaceted concept of MHL. It is especially lacking in assessment of people's health literacy, thus evaluation of MHL based on the vignette interview approach may be incomplete. Furthermore, the vignette interview approach does not provide for a total or subscale score to be generated, nor does it allow for subscales to be included, making it impossible to make individual-level comparisons on each specific attribute of the multifaceted MHL. ${ }^{2}$ Moreover, the questions of the vignette are not independent, with the answer to the first question forming the basis for the answers to the remaining questions, which may not reflect the respondent's true knowledge of the aetiology and treatment of mental disorders. ${ }^{2}$

Third, most previous research has focused on the current situation of MHL and its association with demographics characteristics such as gender, age, education and employment, without noticing the potential impact of MHL on a series of health outcomes including physical, mental and social health. Although improved MHL has been proven to generate improved mental health at the individual level in two intervention studies, ${ }^{24} 25$ whether this could be translated into gains at the population level remains to be seen. ${ }^{26}$ Even less is known about the impact of improved MHL on other domains of health such as physical and social health.

In light of these gaps, in the current study, we administered a 20-item instrument developed by the Chinese Ministry of Health $(\mathrm{MOH})$-the Mental Health Knowledge Questionnaire (MHKQ ${ }^{27}$ - to assess MHL in a representative sample of rural adults living in Liuyang City of Hunan Province, China. Based on the broader definition of MHL laid out by Kusan ${ }^{21}$ and Wei $e t a l,{ }^{22}$ the MHKQ assesses both 'disorder literacy' and 'health literacy' of individuals. The MHKQ was used as a nationwide gold standard to assess the public's MHL, after the '20022010 China Mental Health Work Plan ${ }^{28}$ was issued by the $\mathrm{MOH}$, with the purpose of increasing the public's MHL to $50 \%$ by 2010 . The present study aims to measure the current level of MHL in the rural areas of Liuyang county in Hunan province, and to explore its association with sociodemographics and a series of health outcomes including physical, mental and social health.

\section{METHODS}

\section{Participants}

This was a cross-sectional study. The target population was residents aged 18-60 years who had lived in the rural areas of Liuyang county, Hunan province, for over 6 months. The survey was conducted from November 2010 to August 2011. A multistage cluster-sampling method was adopted to identify participants (figure 1). Two towns (Gaoping and Yong'an) were randomly selected from 33 towns of Liuyang county, and then two villages were randomly selected from each town, followed by two samples randomly selected from each village, leading to a total sampling frame of eight samples. Those who were not living in the areas during the research period, those with difficulty in communication due to serious physical or mental illness and those who were cognitively impaired or actively psychotic, were excluded, leading to a final sample of 2052 residents.

Interviewers visited each household and conducted face-to-face interview with each eligible respondent after 


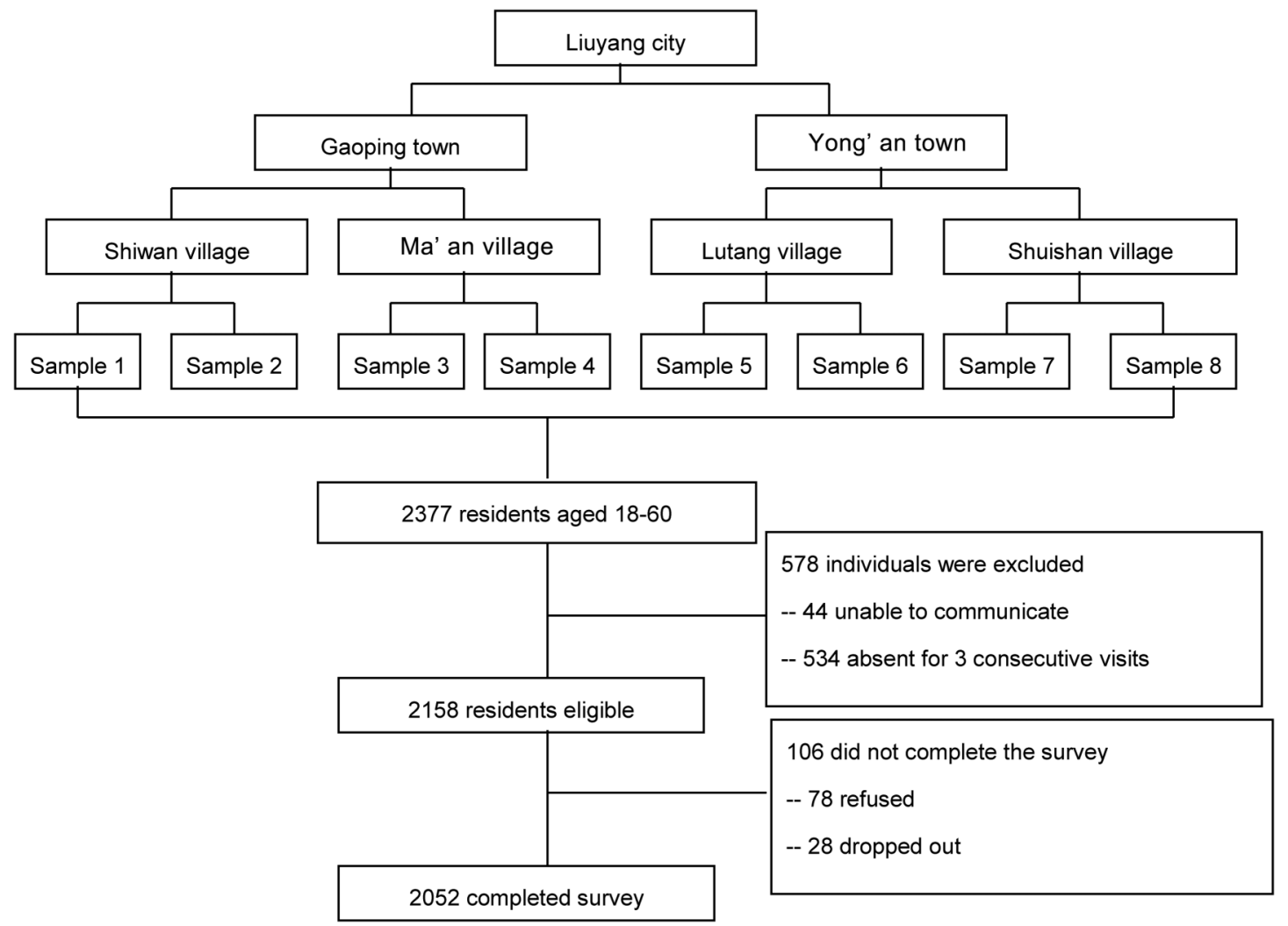

Figure 1 Flow chart of participant enrolment.

obtaining written informed consent. Each interview took approximately $1 \mathrm{~h}$, and each household was reimbursed with small gifts such as kitchen utensils (\$2). Completed surveys were checked by a quality control person to ensure that there were no inconsistencies or missing items.

\section{Instruments}

MHKQ is a 20-item self-report questionnaire developed, in 2009, by the Chinese MOH, to assess public knowledge and awareness of mental health. Items 1-16 are statements about mental health that require respondents to choose 'true', 'false' or 'unknown'; correct responses are 'true' for items $1,3,5,7,8,11,12,15$ and 16, and 'false' for items 2, 4, 6, 9, 10, 13 and 14 . One point is given to each correct answer, with incorrect or unknown responses receiving 0 points. Items $17-20$ ask about whether the respondents have heard of four mental health promotion days, with 1 or 0 points given to those answering 'yes' and 'no', respectively. Past psychometric testing of the scale has reported internal consistency of Cronbach's $\alpha$ coefficients ranging from 0.57 to $0.73^{101129}$ and a 2-week test-retest reliability of 0.68 as measured by intra-class correlation coefficients. ${ }^{29}$ However, there has been inconsistency regarding subscales of the MHKQ, with four ${ }^{11} 29$ and five subscales ${ }^{10}$ being reported in various studies. In the present study, the MHKQ had a Cronbach's $\alpha$ coefficient of 0.61. An exploratory factor analysis yielded a three-factor solution, which covers three aspects of MHL, including knowledge of the characteristics of mental health and mental disorders (items 1, 2, 3, 5, 7, 8, 11, 12, 15 and 16), belief in the epidemiology of mental disorders (items 4, 6, 9, 10, 13 and 14), and awareness of mental health promotion activities (items 17-20), with Cronbach's $\alpha$ coefficients ranging from 0.62 to 0.67 .

The Self-Rated Health Measurement Scale (SRHMS) is a 48 -item scale developed and revised by $\mathrm{Xu}$ et $a \hat{l}^{031}$ to assess self-rated health in hospitalised as well as general populations. It is a comprehensive scale measuring nine domains of health including physical symptoms and organ functions (B1), daily physical activities (B2), physical mobility (B3), psychological symptoms and negative emotions (M1), positive emotions (M2), cognitive function (M3), role activity and social adaptability (S1), social resource and social contact (S2), and social support (S3), which are grouped into three categories in the present study: physical health (BZT), mental health (MZT) and social health (SZT). Each item is rated on a 10-point scale from $1=$ 'extremely poor health' to $10=$ 'extremely good health'. Items $4,5,7,24,25,26$, 27, 28, 29 and 30 are reverse scored and a cumulative score is obtained by adding individual scores. The SRHMS has widespread use in China ${ }^{30} 3233$ and demonstrated good internal consistency in the current study, with a Cronbach's $\alpha$ coefficient of 0.91 for the total scale and $0.80-0.82$ for its three subscales.

The Patient Health Questionnaire (PHQ-9) is one of the most widely used screening tools for depression in 
adults. The 9-item questionnaire is based on criteria for depressive disorders in the Diagnostic and Statistical Manual of Mental Disorders (DSM-IV). ${ }^{34}$ Respondents are asked whether they have been bothered by nine symptoms in the past 2 weeks on a four-point Likert scale from $0=$ 'not at all' to $3=$ 'nearly every day', with higher scores indicating higher depression. A total score is calculated by adding the scores of each item and ranges from 0 to 27. The Chinese version of PHQ-9 has been well validated in multiple studies ${ }^{35-37}$ and showed good internal consistency in the current study, with a Cronbach's $\alpha$ coefficient of 0.81 .

The Generalised Anxiety Disorder Scale (GAD-7) is a 7-item self-report scale developed by Spitzer $e t a l^{38}$ to assess symptoms of and screen for generalised anxiety disorder in primary care settings. Respondents are asked to choose how often they have been bothered by anxiety symptoms, on a four-point Likert scale from $0=$ 'not at all' to $3=$ "nearly every day'. A total score is calculated by adding the scores of each item, and ranges from 0 to 21, with higher scores indicating higher anxiety. The GAD-7 has been widely used and well validated in psychiatric settings $^{39}$ and in general populations, ${ }^{40}$ and demonstrated good internal consistency in the current study, with a Cronbach's $\alpha$ coefficient of 0.88 .

\section{Data analysis}

Data were analysed using STATA software V.12.0. Sociodemographic characteristics of the sample were examined using descriptive statistics. analysis of variance and $\mathrm{t}$ tests were used to compare the MHL score of respondents by gender, age, ethnicity, education, employment, income, marriage and religion, followed by multiple linear regression, to identify the characteristics of respondents that were independently related to the assessed level of MHL. The association between MHL and each of the health outcomes including self-rated mental health, physical health, social health, depression and anxiety were analysed using separate multiple linear regression models adjusted for all demographics.

\section{RESULTS}

\section{Sample characteristics}

A total of 2052 residents completed the questionnaires with a response rate of $95.1 \%$, and their demographic characteristics are summarised in table 1 . The median age of the sample was 42 years with a range of 18-59, with more female $(55.80 \%)$ than male $(44.20 \%)$ participants. Most of the sample were of Han ethnicity $(99.5 \%)$, married $(90.98 \%)$ and non-religious $(90.01 \%)$, with low education (middle school and below: $84.75 \%$ ); $61.11 \%$ of the sample were employed, and over two-thirds had a monthly income of lower than $¥ 300$.

\section{Correct response rate of the MHKQ}

In this sample, the correct response rate of the 20 items ranged from $18.7 \%$ to $94.1 \%$, with a mean correct
Table 1 Sociodemographics of the sample $(\mathrm{N}=2052)$

\begin{tabular}{|c|c|c|}
\hline Characteristics & $\mathbf{n}$ & Per cent \\
\hline \multicolumn{3}{|l|}{ Gender } \\
\hline Male & 907 & 44.20 \\
\hline Female & 1145 & 55.80 \\
\hline \multicolumn{3}{|l|}{ Age (years) } \\
\hline $18-25$ & 227 & 11.06 \\
\hline $26-35$ & 344 & 16.76 \\
\hline $36-45$ & 687 & 33.48 \\
\hline $46-60$ & 794 & 38.69 \\
\hline \multicolumn{3}{|l|}{ Ethnicity } \\
\hline Han & 2042 & 99.51 \\
\hline Non-Han & 10 & 0.49 \\
\hline \multicolumn{3}{|l|}{ Education } \\
\hline Primary school or lower & 814 & 39.67 \\
\hline Middle school & 925 & 45.08 \\
\hline High school and above & 313 & 15.25 \\
\hline \multicolumn{3}{|l|}{ Employment } \\
\hline Unemployed & 797 & 38.84 \\
\hline Employed & 1254 & 61.11 \\
\hline \multicolumn{3}{|l|}{ Income (¥/month/person) } \\
\hline 150 or less & 936 & 45.61 \\
\hline $151-300$ & 475 & 23.15 \\
\hline 300 or greater & 575 & 28.02 \\
\hline \multicolumn{3}{|l|}{ Marital status } \\
\hline Never married & 145 & 7.07 \\
\hline Married/cohabiting & 1867 & 90.98 \\
\hline Divorced/separated/widowed & 40 & 1.95 \\
\hline \multicolumn{3}{|l|}{ Religion } \\
\hline Yes & 205 & 9.99 \\
\hline No & 1847 & 90.01 \\
\hline
\end{tabular}

*Some percentages do not add up to 100 due to missing values.

response rate of $58.1 \%$. The computed MHL score ranged from 0 to 20 and the mean (SD) value was 11.6 (2.9). Awareness rate of the four international mental health awareness days ranged from $18.7 \%$ to $56.8 \%$, with 'International Day Against Drug Abuse and Illicit Trafficking' as the most widely recognised mental health promotion day $(56.8 \%)$. Less than one-quarter of respondents had heard about World Sleep Day (20.3\%), World Mental Health Day (24.5\%) and World Suicide Prevention Day (18.7\%). Of the 16 true-false questions, the items that were most likely to be answered correctly were item 11, 'An optimistic attitude towards life, good inter-personal relationships and healthy living habits are helpful to maintain mental health $(94.1 \%)$; item 5, 'The main content of mental health includes normal intelligence, stable emotion, good mood and harmonious inter-personal relationships' $(87.9 \%)$ and item 1, 'Is mental health an integral part of health?' (87.2\%). Items with the lowest correct response rates were item 2, 'Mental illnesses are caused by ethically (or politically) incorrect thinking' (23.59\%); item 4, 'All mental disorders are due to arousal of violent emotion' (27.34\%) and item 9, 'Psychological/psychiatric disorders can never be prevented' $(41.81 \%)$. The correct response rate of each question is displayed in table 2 . 
Association between sociodemographics and MHL

The mean total score of the sample was 11.63 \pm 2.9 . Table 3 shows the mean MHKQ scores for different demographic groups of participants. There were statistically significant differences in the scores by age, education, employment, income and marital status. Univariate analyses showed that younger age, higher education, being employed, higher income and being single are associated with higher mental health knowledge. Subsequent multiple linear regressions controlling for all demographic factors showed that age, education and income were independently associated with MHL (table 4). Participants of younger age, with higher education and higher income had better MHL. When age increases by 1 year, the MHKQ score decreases by an inconsiderable 0.02 points. When education increases from primary school to middle school and college, MHKQ score increases by 1.38 and 2.69 points, respectively. When income increases from below $¥ 150$ per month to above $¥ 300$ per month, MHKQ score increases by 0.41 points.

\section{Association between MHL and health outcomes}

To explore the association between MHL and health outcomes, we conducted multiple linear regression for
SRHMS, PHQ and GAD independently, adjusting for all demographics. Our results are shown in table 5; MHL was independently positively associated with self-rated general health $(\mathrm{r}=2.31, \mathrm{p}<0.01)$, including physical health $(\mathrm{r}=0.46, \mathrm{p}<0.01)$, mental health $(\mathrm{r}=0.54, \mathrm{p}<0.01)$ and social health $(\mathrm{r}=1.07, \mathrm{p}<0.01)$, regardless of demographics. A one-point increase in MHL led to an increase of 2.31 points in self-rated general health. MHL was also independently negatively associated with depression $(\mathrm{r}=-0.09, \mathrm{p}<0.01)$ and anxiety $(\mathrm{r}=-0.07, \mathrm{p}<0.05)$, regardless of demographics. A one-point increase in MHL led to 0.09-point decrease in depression scores and 0.07 -point decrease in anxiety scores.

\section{DISCUSSION}

This study was conducted to assess MHL in rural areas after the MOH issued the '2002-2010 China Mental Health Work Plan, ${ }^{28}$ which aimed to increase public awareness rate of the characteristics and prevention of mental disorders to $50 \%$ by 2010 . The current study in the rural areas of Liuyang is based on a protocol developed by the Chinese $\mathrm{MOH}$, which produced data on community members' MHL to provide guidance and a basis for future mental health advocacy and education.

Table 2 Correct response rate of Mental Health Knowledge Questionnaire ( $N=2052)$

Item Question [correct response]

Per

cent

16 True-false questions

11 Positive attitudes, good interpersonal relationships and a healthy life style can help maintain mental health [true]

5 Components of mental health include normal intelligence, stable mood, a positive attitude, quality interpersonal relationships and adaptability [true]

1 Mental health is a component of health [true]

7 Psychological or psychiatric services should be sought if one suspects the presence of psychological problems or a mental disorder [true]

16 Mental problems or disorders may occur when an individual is under psychological stress or facing major life events (eg, death of family members) [true]

8 Psychological problems can occur at almost any age [true]

3 Many people have mental problems but do not realise it [true]

15 Individuals with a bad temperament are more likely to have mental problems [true]

13 Psychological problems in adolescents do not influence academic grades [false]

12 Individuals with a family history of mental disorders are at a higher risk for psychological problems and mental disorders [true]

$6 \quad$ Most mental disorders cannot be cured [false]

10 Even for severe mental disorders (eg, schizophrenia), medications should be taken for a given period of time only; there is no need to take them for a long time [false]

14 Middle-aged or elderly individuals are unlikely to develop psychological problems and mental disorders [false]

$9 \quad$ Mental disorders and psychological problems cannot be prevented [false]

$4 \quad$ All mental disorders are caused by external stressors [false]

2 Mental disorders are caused by incorrect thinking [false]

4 Mental health promotion activities

18 Have you heard about the International Day against Drug Abuse and Illicit Drug Trafficking? [true] 56.77

17 Have you heard about International Mental Health Day? [true]

20 Have you heard about World Sleep Day? [true]

19 Have you heard about the International Suicide Prevention Day? [true] 
Table 3 Univariate analysis of the Mental Health Knowledge Questionnaire score

\begin{tabular}{|c|c|c|c|c|}
\hline Item & $\mathbf{n}$ & Mean score & $\mathbf{t} / \mathbf{F}$ & p Value \\
\hline \multicolumn{5}{|l|}{ Total } \\
\hline Gender & 2052 & 11.63 & & \\
\hline Male & 907 & 11.74 & & \\
\hline Female & 1145 & 11.54 & 1.58 & 0.11 \\
\hline \multicolumn{5}{|l|}{ Nationality } \\
\hline Han & 2042 & 11.62 & & \\
\hline Non-Han & 10 & 12.8 & -1.26 & 0.21 \\
\hline \multicolumn{5}{|l|}{ Age (years) } \\
\hline $18-25$ & 227 & 12.48 & & \\
\hline $26-35$ & 344 & 12.54 & & \\
\hline $36-45$ & 687 & 11.58 & & \\
\hline $46-60$ & 794 & 11.01 & 29.97 & $<0.01$ \\
\hline \multicolumn{5}{|l|}{ Education } \\
\hline Primary school or less & 814 & 10.45 & & \\
\hline Middle school & 925 & 12.06 & & \\
\hline College and above & 313 & 13.42 & 151.88 & $<0.01$ \\
\hline \multicolumn{5}{|l|}{ Employment } \\
\hline Unemployed & 797 & 11.41 & & \\
\hline Employed & 1254 & 11.76 & 2.66 & 0.01 \\
\hline \multicolumn{5}{|l|}{ Income ( $¥ /$ month/person) } \\
\hline 150 or less & 936 & 11.29 & & \\
\hline $151-300$ & 475 & 11.81 & & \\
\hline 300 or greater & 575 & 12.07 & 13.84 & $<0.01$ \\
\hline \multicolumn{5}{|l|}{ Marital status } \\
\hline Married/cohabited & 1867 & 11.58 & & \\
\hline Divorced/separated/widowed & 40 & 11.35 & & \\
\hline Never married & 145 & 12.37 & 5.02 & 0.01 \\
\hline \multicolumn{5}{|l|}{ Religion } \\
\hline Yes & 205 & 11.42 & & \\
\hline No & 1847 & 11.65 & -1.04 & 0.30 \\
\hline
\end{tabular}

The mean overall correct response rate of MHL identified in this study is $58 \%$, suggesting that the goal of '2002-2010 China Mental Health Work Plan' was achieved in the rural areas of Liuyang. However, this rate is lower than those reported in urban areas of China such as Shanghai $(72 \%),{ }^{10}$ Guangzhou $(60.2 \%),{ }^{41}$ Changsha $(68.5 \%)^{11}$ and Xi'an $(70.3 \%){ }^{42}$ This may be explained by the less developed economy in rural areas of China. MHL in less developed rural areas is in need of improvement through population wide interventions and also through individual training programmes. ${ }^{26}$

Compared to the $57 \%$ awareness rate of the International Day Against Drug Abuse and Illicit Trafficking, the awareness rates of World Mental Health Day, World Suicide Prevention Day and World Sleep Day are much lower. The reason may be that the government has put much greater emphasis on drug abuse and trafficking, due to its greater potential to threaten and endanger social safety and stability compared to other mental health problems such as sleep disorders and suicide. Promotion efforts on the other 3 days need to be strengthened by the government to increase public awareness of these issues and popularise knowledge about them. Most people (over 70\%) held misbeliefs about the aetiology of mental illness, trusting that ethically incorrect thinking or the arousal of violent emotions causes mental illnesses. These misattributions are consistent with reports in previous studies in China, ${ }^{10}{ }^{11}$ implying that misattribution of mental illness is prevalent throughout the country. The misconception of the causes of mental illness may be an important antecedent for the pervasive negative attitudes towards mentally ill patients. Future targeted intervention may benefit by educating the public on accurate recognition of causes of mental illness.

Significant differences in the MHL score between different demographic groups of respondents were found in the current study. Multivariate linear regression indicated that younger age, higher education and higher income were the most robust factors associated with higher MHL. These results are consistent with previous studies. ${ }^{10-12}$ Higher education, as an indicator of higher health literacy, can be seen as an indirect indicator of MHL and thus is associated with higher MHL. Higher income, usually accompanied with higher education, is also related to higher MHL. Interestingly, being younger, rather than being older, is associated with higher MHL. The reason may be related to the different social political environments that different generations have experienced. During the Chinese Cultural 
Table 4 Multiple linear regression of Mental Health Knowledge Questionnaire scale scores

\begin{tabular}{|c|c|c|c|c|c|}
\hline Variable & Coefficient & $\begin{array}{l}\text { Standard } \\
\text { coefficient }\end{array}$ & $\mathbf{t}$ & $p>|t|$ & $95 \% \mathrm{Cl}$ \\
\hline Age (continuous) & -0.02 & 0.01 & -3.51 & $<0.001^{\star \star}$ & $(-0.04$ to -0.01$)$ \\
\hline \multicolumn{6}{|l|}{ Gender } \\
\hline \multicolumn{6}{|l|}{ Male (ref) } \\
\hline Female & -0.00 & 0.14 & -0.00 & 0.997 & $(-0.27$ to 0.27$)$ \\
\hline \multicolumn{6}{|l|}{ Nationality } \\
\hline \multicolumn{6}{|l|}{ Han (ref) } \\
\hline Non-Han & 1.34 & 0.87 & 1.53 & 0.125 & $(-0.37$ to 3.06$)$ \\
\hline \multicolumn{6}{|l|}{ Education } \\
\hline \multicolumn{6}{|l|}{ Primary school or less (ref) } \\
\hline Middle school & 1.38 & 0.14 & 9.53 & $<0.001^{\star *}$ & (1.10 to 1.66$)$ \\
\hline College and above & 2.69 & 0.21 & 13.03 & $<0.001^{\star *}$ & (2.41 to 3.23 ) \\
\hline \multicolumn{6}{|l|}{ Employment } \\
\hline \multicolumn{6}{|l|}{ Unemployed (ref) } \\
\hline Employed & -0.22 & 0.14 & -1.60 & 0.109 & $(-0.49$ to 0.05$)$ \\
\hline \multirow{2}{*}{\multicolumn{6}{|c|}{ Income ( $¥ /$ month/person) }} \\
\hline \multicolumn{4}{|l|}{150 or less (ref) } & & \\
\hline $151-300$ & 0.25 & 0.16 & 1.63 & 0.104 & $(-0.05$ to 0.56$)$ \\
\hline 300 or greater & 0.41 & 0.15 & 2.73 & $0.006^{* *}$ & (0.12 to 0.70$)$ \\
\hline \multicolumn{6}{|l|}{ Marital status } \\
\hline \multicolumn{6}{|l|}{ Married/cohabited (ref) } \\
\hline Divorced/separated/widowed & 0.13 & 0.26 & 0.49 & 0.624 & $(-0.39$ to 0.65$)$ \\
\hline Never married & 0.58 & 0.53 & 1.09 & 0.276 & $(-0.46$ to 1.63$)$ \\
\hline \multicolumn{6}{|l|}{ Religion } \\
\hline Yes (ref) & & & & & \\
\hline No & -0.04 & 0.21 & -0.21 & 0.837 & $(-0.45$ to 0.36$)$ \\
\hline
\end{tabular}

Revolution (1966-1976), education was not valued by the Chinese government, leading to many people not receiving an education when they were young. It was not until 1986, when the government introduced the 9-year compulsory education policy, that education became available to all citizens in the nation. Since then, the educational level has increased throughout China. Hence, the negative correlation found between age and MHL may reflect China's political history.
The positive impact of high MHL on a series of health outcomes including physical, mental and social health, is established in the present study. The result that people with higher MHL also enjoy better physical and social health is a novel finding that has not been reported in the existing literature. The mechanism behind this association may be related to the improved help-seeking behaviour that has been widely documented among people with higher MHL, ${ }^{16-19}$ which may

Table 5 Association between mental health literacy (MHL) and each health outcome (Self-Rated Health Measurement Scale (SRHMS), Patient Health Questionnaire (PHQ) and Generalised Anxiety Disorder Scale (GAD)) using multiple linear regression models adjusted for demographics $\dagger$

\begin{tabular}{|c|c|c|c|c|c|}
\hline Health outcomes & Coefficient & $\begin{array}{l}\text { Standard } \\
\text { coefficient }\end{array}$ & $\mathbf{t}$ & $p>|t|$ & $95 \% \mathrm{Cl}$ \\
\hline \multicolumn{6}{|l|}{ SRHMS } \\
\hline Physical health & 0.46 & 0.15 & 3.14 & $0.002^{\star *}$ & $(0.17$ to 0.75$)$ \\
\hline Mental health & 0.54 & 0.17 & 3.20 & $0.001^{\star *}$ & (0.21 to 0.87$)$ \\
\hline Social health & 1.07 & 0.14 & 7.56 & $<0.001^{\star *}$ & (0.80 to 1.35$)$ \\
\hline Total health & 2.31 & 0.40 & 5.79 & $<0.001^{\star \star}$ & (1.53 to 3.09 ) \\
\hline \multicolumn{6}{|l|}{$\mathrm{PHQ}$} \\
\hline Depression & -0.09 & 0.03 & -2.81 & $0.005^{\star \star}$ & $(-0.15$ to -0.03$)$ \\
\hline \multicolumn{6}{|l|}{ GAD } \\
\hline Anxiety & -0.07 & 0.03 & -2.50 & $0.013^{*}$ & $(-0.13$ to -0.02$)$ \\
\hline
\end{tabular}


lead to increased general health outcomes including physical health and social health. The finding that increased MHL is associated with increased general health provides strong motivation to enhance population MHL through a series of educational campaigns.

One limitation of the present study is the unfavourable internal consistency of the 20-item MHKQ scale (Cronbach's $\alpha=0.61$ ), which is in keeping with previous studies showing weak to acceptable internal consistency. ${ }^{10}{ }^{11}{ }^{29}$ However, this may be due to the fact that for knowledge scales measuring various factors of a construct, knowing the correct answer of one item does not necessarily lead to the right recognition of another item, thus a very high internal consistency may not be expected. Indeed, as a standardised government document developed by the Chinese $\mathrm{MOH}$ to assess the public knowledge and awareness of mental health, the MHKQ has been popularised as a national gold standard to compare MHL across the country. The purpose of the present study is not to test the psychometric properties of the MHKQ, but to compare the level of MHL in the rural areas of Liuyang with other parts of China. As a result, the psychometric properties of the MHKQ are not of primary importance, given that the scale is comparable across different studies.

Another limitation is the cross-sectional design of the study, which precludes evaluation of temporal sequence of MHL and health outcomes, and causal relationships cannot be determined. However, according to the scale's design, the SRHMS assesses self-rated health in the past 4 weeks, ${ }^{30}{ }^{31}$ the $\mathrm{PHQ}^{34}$ and $\mathrm{GAD}^{38}$ measure depression and anxiety in the past 2 weeks, while the MHKQ measures an individual's lifetime knowledge and belief about mental health, which may take root in a person's early life. We could infer a potential causal relationship between MHL and health outcomes.

A third limitation is a lack of comparison between MHKQ and other multifaceted scales intending to measure MHL. The fact that MHKQ is developed and used only in China makes it difficult to compare the psychometric properties of the Chinese version of MHKQ with scales used in populations outside China, since psychometric testing is also sample dependent. ${ }^{43}$ Future research may benefit from applying the English version of the MHKQ together with other scales in the same study to compare its feasibility and psychometric properties.

In conclusion, MHL in the rural areas of Liuyang is lower than that reported in urban areas of China. More efforts are needed to increase public awareness of mental health promotion activities and more population-level intervention programmes are needed to educate the public about the correct attribution of mental disorders. Younger age, higher education and higher income are the three strongest factors related to higher MHL, so educational interventions may need to be cohort specific, with special attention paid to those who are older, poorer and less educated. Higher MHL is related to better general health including physical, mental and social health, suggesting that improvement of MHL may enhance overall population health, warranting future exploration.

\section{Ethical statement}

This study was approved by the Institutional Review Boards of the School of Public Health of Central South University, and was performed in accordance with the ethical standards laid down in the 1964 Declaration of Helsinki and its later amendments. All participants in the present study gave their informed consent prior to their inclusion in the study. Details that might disclose the identity of the subjects under study were omitted and all data collected were unidentified.

Acknowledgements The authors would like to thank the village cadres for guiding us to visit each household in the rural areas of Liuyang county, Hunan province, China.

Contributors SX and LZ conceived and designed the study. YY, ZL, MH, XL and JPY collected the data. YY and $Z \mathrm{~L}$ analysed and interpreted the data. $Y Y$ drafted the article, while $S X, L Z, M H, Z L, X L$ and JPY critically revised it for intellectual content. All the authors gave final approval to the version submitted for publication.

Funding This study was funded by the National Science and Technology Support Program, China (Number 2009BAl77B01; Number 2009BAI77B08) and the Hunan Provincial Innovation Foundation for Postgraduate (number CX2015B058).

Competing interests None declared.

Patient consent Obtained.

Ethics approval Institutional Review Boards of the School of Public Health of Central South University.

Provenance and peer review Not commissioned; externally peer reviewed.

Data sharing statement All data underlying the findings in our study are freely available on request; please contact YY at youxiang8864@163.com for the dataset and analysis commands.

Open Access This is an Open Access article distributed in accordance with the Creative Commons Attribution Non Commercial (CC BY-NC 4.0) license, which permits others to distribute, remix, adapt, build upon this work noncommercially, and license their derivative works on different terms, provided the original work is properly cited and the use is non-commercial. See: http:// creativecommons.org/licenses/by-nc/4.0/

\section{REFERENCES}

1. Jorm AF, Korten AE, Jacomb PA, et al. "Mental health literacy": a survey of the public's ability to recognise mental disorders and their beliefs about the effectiveness of treatment. Med J Aust 1997;166:182-6.

2. O'Connor M, Casey L, Clough B. Measuring mental health literacy-a review of scale-based measures. J Ment Health 2014;23:197-204.

3. Olsson DP, Kennedy MG. Mental health literacy among young people in a small US town: recognition of disorders and hypothetical helping responses. Early Interv Psychiatry 2010;4:291-8.

4. Coles ME, Coleman SL. Barriers to treatment seeking for anxiety disorders: initial data on the role of mental health literacy. Depress Anxiety 2010;27:63-71.

5. Okuyama T, Nakane $\mathrm{Y}$, Endo $\mathrm{C}$, et al. Mental health literacy in Japanese cancer patients: ability to recognize depression and preferences of treatments-comparison with Japanese lay public. Psychooncology 2007;16:834-42.

6. Loureiro LM, Jorm AF, Oliveira RA, et al. Mental health literacy about schizophrenia: a survey of Portuguese youth. Early Interv Psychiatry 2015;9:234-41. 
7. Wong DF, Lam AY, Poon A, et al. Gender differences in mental health literacy among Chinese-speaking Australians in Melbourne, Australia. Int J Soc Psychiatry 2012;58:178-85.

8. Cotton SM, Wright A, Harris MG, et al. Influence of gender on mental health literacy in young Australians. Aust N Z J Psychiatry 2006;40:790-6.

9. Koyama T, Tachimori $\mathrm{H}$, Sawamura $\mathrm{K}$, et al. Mental health literacy of autism spectrum disorders in the Japanese general population. Soc Psychiatry Psychiatr Epidemiol 2009;44:651-7.

10. Wang J, He Y, Jiang Q, et al. Mental health literacy among residents in Shanghai. Shanghai Arch Psychiatry 2013;25:224-35.

11. Peng Y, Wang XL, Li PF, et al. Mental health literacy in Changsha, China. Shanghai Arch Psychiatry 2011;23:353-9(in Chinese).

12. Furnham A, Annis J, Cleridou K. Gender differences in the mental health literacy of young people. Int $J$ Adolesc Med Health 2014;26:283-92.

13. Chiang JCS, Chow ASY, Chan RCK, et al. Pathway to care for patients with first-episode psychosis in Hong Kong. Hong Kong $J$ Psychiatr 2005; 15:18-23.

14. Li H. Mental health literacy, stigma, and early intervention. Asian $J$ Psychiatr 2012;5:209-10.

15. Loo PW, Furnham A. Public knowledge and beliefs about depression among urban and rural Chinese in Malaysia. Asian $\mathrm{J}$ Psychiatr 2012;5:236-45.

16. Jorm AF, Kelly CM, Wright $A$, et al. Belief in dealing with depression alone: results from community surveys of adolescents and adults. $J$ Affect Disord 2006;96:59-65.

17. Mendenhall AN. Predictors of service utilization among youth diagnosed with mood disorders. J Child Fam Stud 2012;21: 603-11.

18. Mendenhall AN, Fristad MA, Early TJ. Factors influencing service utilization in mood symptom severity in children with mood disorders: Effects of multifamily psychoeducation groups. J Consult Clin Psychol 2009;77:463-73.

19. Jorm AF, Christensen H, Medway J, et al. Public belief systems about the helpfulness of interventions for depression: associations with history of depression and professional help-seeking. Soc Psychiatry Psychiatr Epidemiol 2000;35:211-19.

20. Kusan S. Reconstructing the mental health narrative: bio-cultural grammar, symbolic text translation and the coalescence of meanings. Int J Learn 2012;18:442-61.

21. Kusan S. Dialectics of mind, body, and place: groundwork for a theory of mental health literacy. SAGE Open 2013;3:1-16.

22. Wei Y, Hayden JA, Kutcher S, et al. The effectiveness of school mental health literacy programs to address knowledge, attitudes and help seeking among youth. Early Interv Psychiatry 2013;7:109-21.

23. Jorm AF, Christensen H, Griffiths KM. The public's ability to recognize mental disorders and their beliefs about treatment: changes in Australia over 8years. Aust N Z J Psychiatry 2006;40:36-41.

24. Kitchener BA, Jorm AF. Mental health first aid training: review of evaluation studies. Aust N Z J Psychiatry 2006;40:6-8.

25. Christensen H, Griffiths KM, Jorm AF. Delivering interventions for depression by using the internet: randomised controlled trial. BMJ 2004;328:265.
26. Jorm AF, Barney LJ, Christensen $\mathrm{H}$, et al. Research on mental health literacy: what we know and what we still need to know. Aust $N$ Z J Psychiatry 2006;40:3-5.

27. Health CMo. Survey evaluation protocol of mental health work from the General Office of the Ministry of Health (MOHCDC[2010] Number 24). Beijing: Ministry of Health. http://www.gov.cn/gzdt/201003/08/ content_1550552.htm (accessed 1 Aug 2013) (in Chinese).

28. Ministries of Health, Civil Affairs, Public Security, et al. Mental Health Work Plan of China (2002-2010). Shanghai Arch Psychiatry 2003:15:125-8.

29. Zhong BL, Chen HH, Xiao SY, et al. Reliability and validity of mental health knowledge questionnaire for middle school students. Chin J Sch Health 2011;32:49-50.

30. Xu J, Wang BH, Hu MY, et al. The Development and evaluation of Self-Rated Health Measurement Scale-prior test version. Chin J Behav Med Sci 2000;9:65-8.

31. Xu J, Guo R, Liu YS, et al. The Study of Responsiveness on Self-rated Health Measurement Scale (the Revised Version 1.0). Chin J Health Stat 2003;20:272-5.

32. Xu J, Zhang J, Feng L, et al. Self-rated health of population in Southern China: association with socio-demographic characteristics measured with multiple-item Self-Rated Health Measurement Scale. BMC Public Health 2010;10:393.

33. Xu Y, Xue ZM. A Comparative Study of SCL-90 and Self-rated Health Measurement Scale in College Students. Chin J Clin Psychol 2003;3:232.

34. Spitzer RL, Kroenke K, Williams JB. Validation and utility of a self-report version of PRIME-MD: the PHQ primary care study. Primary Care Evaluation of Mental Disorders. Patient Health Questionnaire. JAMA 1999;282:1737-44.

35. Wang W, Bian Q, Zhao Y, et al. Reliability and validity of the Chinese version of the Patient Health Questionnaire (PHQ-9) in the general population. Gen Hosp Psychiatry 2014;36:539-44.

36. Chen TM, Huang FY, Chang C, et al. Using the PHQ-9 for depression screening and treatment monitoring for Chinese Americans in primary care. Psychiatr Serv 2006;57:976-81.

37. Chen S, Chiu H, Xu B, et al. Reliability and validity of the PHQ-9 for screening late-life depression in Chinese primary care. Int $J$ Geriatr Psychiatry 2010;25:1127-33.

38. Spitzer RL, Kroenke K, Williams JB, et al. A brief measure for assessing generalized anxiety disorder: the GAD-7. Arch Intern Med 2006; 166:1092-7.

39. Beard C, Bjorgvinsson T. Beyond generalized anxiety disorder: psychometric properties of the GAD-7 in a heterogeneous psychiatric sample. J Anxiety Disord 2014;28:547-52.

40. Lowe B, Decker O, Muller S, et al. Validation and standardization of the Generalized Anxiety Disorder Screener (GAD-7) in the general population. Med Care 2008:46:266-74

41. Hu HY, Yan YZ, Chen LM, et al. Knowledge about mental health and attitude to mental disorder in urban and rural residents in Guangzhou. Chin Mental Health J 2012;26:30-5.

42. Liang XP, Zhu XJ, Liu T, et al. Cross-sectional survey on awareness of mental health knowledge among communities in Xi'an. China $J$ Health Psychol 2011;25:1200-2.

43. Yu Y, Shiu CS, Yang JP, et al. Factor analyses of a social support scale using two methods. Qual Life Res 2015;24:787-94. 